\title{
Radiation-induced mixing and demixing behavior in metallic multilayers exhibiting limited solid miscibility
}

Madhavan Radhakrishnan ${ }^{1}$, Thomas Nizolek ${ }^{2},{\mathrm{Nan} \mathrm{Li}^{2} \text {, Yongqiang Wang }}^{2}$, Mukesh Bachhav ${ }^{3}$, Boopathy Kombaiah $^{3}$ and Osman Anderoglu ${ }^{1}$

${ }^{1}$ University of New Mexico, United States, ${ }^{2}$ Los Alamos National Laboratory, United States, ${ }^{3}$ Idaho National Laboratory, United States

Next generation nuclear reactors demand structural materials that withstand large irradiation doses at elevated temperatures. One of the ways to improve the irradiation tolerance in materials is to introduce a large fraction of interfaces. Past studies have shown that nanostructured composites show remarkable resistance to radiation damage due to interfaces-driven defects absorption ${ }^{1}$. In this talk, radiation behavior of nanolayered zirconium/niobium and copper/niobium composites subjected to high irradiation doses will be discussed. The nanocomposites were synthesized in bulk form by accumulative roll bonding, a severe plastic deformation technique. Both systems show limited solubility at the irradiation temperature and exhibit a positive heat of mixing. The multilayers were subjected to self-ion irradiated doses of 140-200 dpa (displacements per atom) with $\mathrm{Zr}^{2+}$ and $\mathrm{Cu}^{2+}$ ions. Cross-sectional transmission electron microscopy examination indicates that zirconium/niobium multilayers with layer thicknesses spanning 15-80nm have undergone chemical mixing of layers and grain growth beneath the irradiated surface. On the other hand, all copper/niobium multilayers showed remarkable stability of layers and sharp interfaces up to 200 dpa dose, except for the thickening of copper and niobium layers in the maximum dose region. The observed mixing/demixing behavior in multilayers is attributed to chemically-driven interphase diffusion events within the thermal spikes. The experimental results validates that the chemical distribution under ion-radiation is sensitive to liquid-phase solubilities ${ }^{2,3}$. The talk would emphasis the dependence of final microstructure and solute partition on the competition between migration of atom species triggered by the irradiation-induced thermal spikes and the thermally-activated radiation enhanced diffusion.

Acknowledgements: This work is supported by Nuclear Energy University Program (NEUP) (grant \# DENE0008656) and NRC faculty development grant (NRC-HQ-60-17-G-0007).
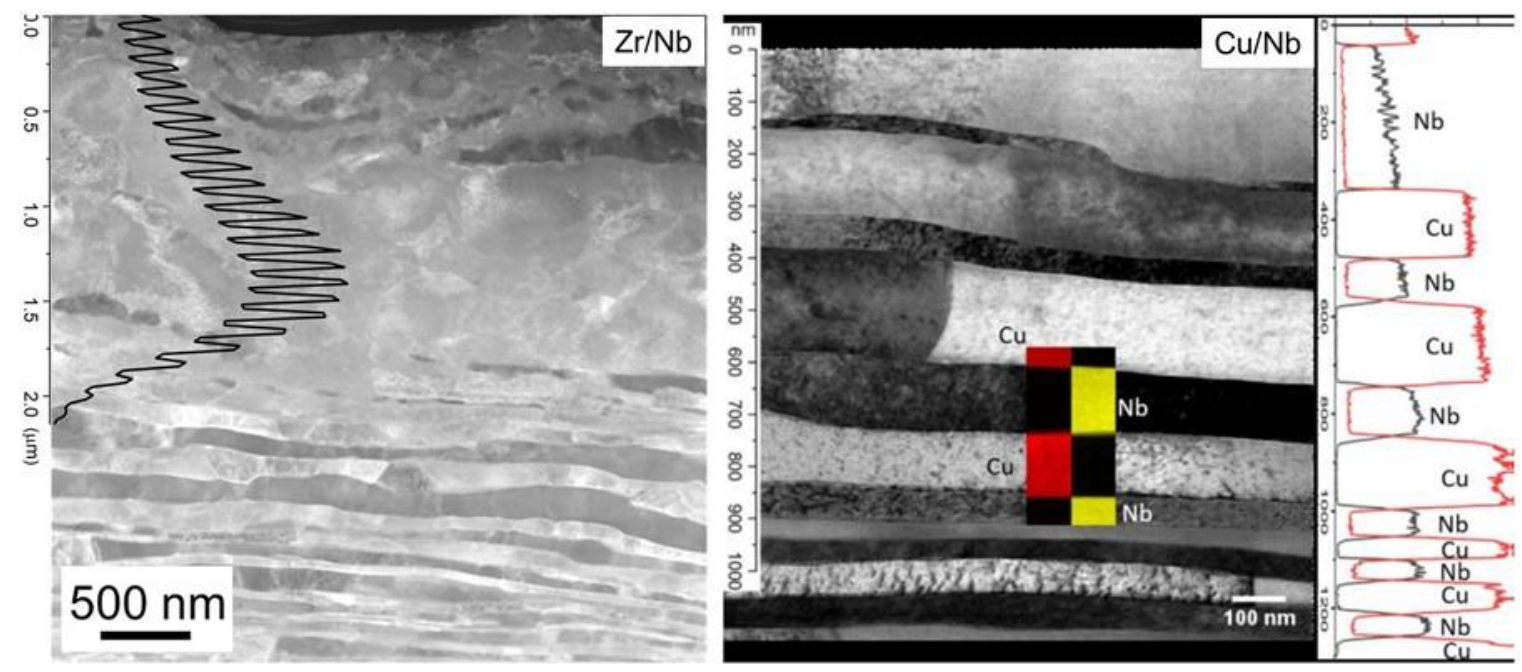

Figure 1. Sub-surface irradiated microstructures in $\mathrm{Zr} / \mathrm{Nb}$ and $\mathrm{Cu} / \mathrm{Nb}$ multilayers after the dose of $140 \mathrm{dpa}$ and $100 \mathrm{dpa}$, respectively. The extent of chemical mixing and layer fragmentation, corresponding to irradiated dose levels, are evident in $\mathrm{Zr} / \mathrm{Nb}$. The interface stability and layer thickening is apparent in $\mathrm{Cu} / \mathrm{Nb}$. 


\section{References}

1. A Misra, JP Hirth, RG Hoagland, Acta Materialia, 53, 2005, p. 4817

2. RS Averback, D Peak, LJ Thompson, Applied Physics A, 39, 1986, p. 59.

3. L Zhang, M Demkowicz, Acta Materialia, 76, 2014, p. 135. 